\title{
DISPENSAÇÃO DE ANTIDEPRESSIVOS CONTROLADOS PELA PORTARIA 344/1998, EM FEIRA DE SANTANA - BA NO PERÍODO DA PANDEMIA DO COVID-19
}

\author{
DISPENSATION OF ANTIDEPRESSANTS CONTROLLED BY ORDINANCE 344/1998, \\ IN FEIRA DE SANTANA - BA DURING THE COVID-I9 PANDEMIC PERIOD
}

\author{
Diego Rafael Sampaio Lima ${ }^{1}$ \\ Monique Barbosa Moura ${ }^{2}$ \\ Reinaldo de Almeida Oliveira 3 \\ Rodrigo Inácio Novais de Oliveira ${ }^{4}$ \\ Vinícius Mendes de Souza Carneiro ${ }^{5}$
}

RESUMO: Este estudo investigou a prevalência $e$ o aumento da venda de medicamentos antidepressivos na cidade de Feira de Santana-BA, no período de março de 2019 a fevereiro de 2021, fazendo recortes temporais entre março/2019 e fevereiro de 2020 como período pré-pandemia e março de 2020 a fevereiro de 2021 como período de pandemia. Os dados mostram que medicamentos como a Amitriptilina $25 \mathrm{mg}$, fluoxetina 2omg, escitalopram Io $\mathrm{mg}$ e a sertralina $50 \mathrm{mg}$ que já eram bem utilizados como medicamentos antidepressivos, mas que durante a pandemia esse número cresceu. Os fatores que podem estar associados a esse crescimento são $o$ isolamento e o distanciamento social, a insegurança financeira como consequência de lockdown e o medo da morte causada pelo vírus foram impostos pela pandemia de covid-19. Os resultados obtidos contribuíram também para a avaliação das concentrações e classes de medicamentos dispensados entre as datas citadas acima, demostrando a análise entre as vendas sob dispensação de medicamentos antidepressivos.

Palavras-chave: Covid-19. Depressão. Pandemia. Saúde mental. Antidepressivo.

ABSTRACT: This study investigated the prevalence and increase in the sale of antidepressant pills in the city of Feira de Santana-BA, from March 2019 to February 2021, making timelines between March/2019 and February 2020 as a pre-pandemic period and March 2020 to February 202I as a pandemic period. The data show that medications such as Amitriptyline 25mg, Fluoxetine 20mg, Escitalopram Iomg and Sertraline 5omg were already well used as antidepressant drugs, but during the

\footnotetext{
${ }^{1}$ Universidade de Salvador- Unifacs. Formando em farmácia. E-mail: diegorafaelsampaior2345@hotmail.com

${ }^{2}$ Universidade de Salvador- Unifacs. Formanda em farmácia. E-mail: nique.moura@hotmail.com

${ }^{3}$ Universidade de Salvador- Unifacs. Formanda em farmácia. E-mail: reialmeida77@hotmail.com

${ }^{4}$ Universidade de Salvador- Unifacs. Formanda em farmácia. -mail: oliveira.rodrigonovais@gmail.com

${ }^{5}$ Universidade Salvador-UNIFACS, Bahia, Brasil. Mestre em Zoologia. E-mail:

vmscarneiro@outlook.com
} 
pandemic, this number grew. Factors that may be associated with this growth are isolation and social distancing, financial insecurity because of the lockdown and the fear of death caused by the virus were caused by the covid-I9 pandemic. The results obtained also contributed to the evaluation of the measures of medications dispensed between the dates mentioned above, demonstrating an analysis between sales under dispensing of antidepressant medications.

Keyboards: Covid-ı. Depression. Pandemic. Mental health. Antidepressant.

\section{INTRODUÇÃO}

O Covid-19 (novo corona vírus), surgiu destacando-se como um vírus desconhecido que deixou toda população aflita com seu surgimento, atingido todos os países do mundo.

De acordo com a OMS - Organização Mundial de Saúde (202I) um pouco mais 220 milhões de pessoas foram acometidas com essa terrível doença e mais de 4,5 milhões vieram a óbito.

Algumas das principais e emergenciais medidas para se combater esse vírus até então desconhecido foi o uso da máscara, lavar as mãos com água e sabão, usar álcool 70\%, distanciamento e isolamento social.

Em consequência desse isolamento social, a população em geral com medo pois se tratava de uma doença desconhecida, recorreram a notícias televisionadas e redes sociais e se sentiram apavoradas, algumas com medo de morrer e outras com medo de ficarem desempregadas, pois muitas empresas foram obrigadas a fecharem por imposição dos governantes. Com tudo isso a busca por alívio dos sintomas de ansiedade e angústia, fizeram com que muitos procurassem ajuda através dos medicamentos.

Conforme o boletim Pílula Farmacêutica da Faculdade de Ciências Farmacêuticas de Ribeirão Preto (FCFRP) elaborado pelo Jornal da USP, com supervisão da professora Regina Célia Garcia de Andrade.

Os antidepressivos dessa classe mais populares são a fluoxetina, citalopram, paroxetina, sertralina, fluvoxamina e escitalopram. $O$ efeito antidepressivo ocorre pelos inibidores seletivos da recaptação da serotonina, que agem impedindo a retirada da serotonina da fenda sináptica, local onde esse neurotransmissor exerce suas ações. Desse modo, a serotonina permanece 
disponível por mais tempo, causando melhora no humor dos pacientes. (COLTRI, 2019)

Os medicamentos citados acima se usados excessivamente e sem orientação médica e farmacêutica geram efeitos colaterais como cefaleia, gastrointestinais, dificuldade na coordenação motora, distúrbios no sono e no nível de energia e em alguns casos, dependência química e disfunção sexual.

A depressão foi considerada pela Organização Mundial da Saúde (OMS) (202I) como o "mal do século".

Segundo a Revista de Psicofisiologia:

A depressão é um distúrbio afetivo cujos principais sintomas são: tristeza, pessimismo, baixa autoestima, e em casos mais graves e sem acompanhamento, pode levar à morte (em sua maioria, por suicídio) e necessita de diagnóstico médico e tratamento adequados. (JÚNIOR et. al., I998, p.II)

A classe de pessoas mais afetadas durante a pandemia foram os idosos que eram acostumados a ter seus familiares por perto, a viajar, a participar de programas em grupos de melhor idade, de repente foram impedidos desse convívio.

De acordo com a Organização Mundial da Saúde (2002), em estudo epidemiológico a prevalência de depressão ao longo da vida no Brasil está em torno de $15,5 \%$ e a faixa etária mais comum do aparecimento é o final da $3^{\underline{a}}$ década da vida, mas pode começar em qualquer idade.

De surgimento emergencial na saúde física e mental o corona vírus veio fazendo história. A evolução do vírus é de forma rápida e fácil contágio, foram assim adotadas pelos governos municipais as medidas de restrição pública como o isolamento social e a quarentena, essas medidas juntamente com o medo de ser infectado pela doença gerou uma grande sobrecarga e fragilidade emocional na população.

Os impactos gerados na saúde mental vivido pela população de modo geral e pelos profissionais da saúde que direta ou indiretamente enfrentava os desafios, enquanto as medidas cabíveis vinham sendo utilizadas no intuito de vir a amenizar o sofrimento mental da população (GUIMARÃES, 2020).

No que se refere ao artigo, os casos de depressão aumentaram 90\% e o número de 
pessoas que relataram sintomas como crise de ansiedade e estresse agudo mais que dobrou entre os meses de março e abril deste ano.

Conforme o IBICT (Instituto Brasileiro de Informação em Ciência e Tecnologia) (SCHEINKMAN, 202I) há relatos de alterações psicopatológicas provocadas por outros coronavírus, como o SARS (Severe Acuty Respiratory Syndrome) e a MERS (Middle East Respiratory Syndrome). Pessoas que foram acometidas por tais vírus, informaram sintomas psiquiátricos como Transtorno de Estresse Pós-Traumático (TEPT), depressão, transtorno de pânico, e TOC. Considerando tais evidências, pesquisadores acreditam que o novo coronavírus também possa provocar alterações neurológicas.

Uma pesquisa feita pela Universidade de São Paulo (USP) (COLTRI, 2019) afirma que o Brasil é o país com maiores episódios de depressão durante a pandemia, onde as principais causas que contribuíram para tal colocação são o isolamento social e o avanço da doença, sendo que com o isolamento vem o desemprego e retardo do desenvolvimento pessoal e profissional.

Pensando nisto, em outubro de 2020 a Prefeitura de Feira de Santana, Bahia informou que pacientes terão atendimento especializado no pós-covid, que, segundo o site oficial do órgão "continuam sofrendo com os sintomas da doença". A infectologista Melissa Falcão, coordenadora do comitê de enfrentamento ao corona vírus da cidade, relatou que dentre as alterações psicológicas, encontra-se alteração de humor, depressão e insônia (2021).

O objetivo do presente estudo foi avaliar o aumento do uso de medicações psicotrópicos com ação antidepressiva, após o início da pandemia (março de 2020 a fevereiro de 2021). Tendo em vista os efeitos adversos que tais substâncias podem provocar no organismo e os principais motivos que levaram as pessoas a buscarem profissionais especializados.

\section{METODOLOGIA}

O artigo é um estudo quantitativo, que compara dados dos medicamentos 
vendidos na cidade de Feira de Santana - Bahia, no período de março de 2019 a fevereiro de 2020 e este, é considerado por esta pesquisa o período pré-pandemia. Logo após, foram colhidos os dados do período de pandemia que nesta pesquisa são considerados todos os casos positivos seguintes ao surgimento do primeiro caso de Covid-ı que conforme SESAB (Secretaria da Saúde do Estado da Bahia) o vírus SARS-COV-2 chegou à Feira de Santana - Bahia, em o6 de março de 2020 com uma mulher que voltava de uma viagem à Itália, sendo este o nono caso confirmado no Brasil (2021). O período da pesquisa se estendeu até o mês de fevereiro de 2021.

Os medicamentos dispensados e selecionados se caracterizam como industrializados e suas classes terapêuticas incluem: Inibidores da recaptação de norepinefrina, Inibidores seletivos da recaptação de serotonina e Inibidores da monoaminoxidase que se enquadram como medicamentos antidepressivos (HILALDANDAN, 2014).

Foram excluídos da pesquisa medicamentos os quais não houve venda em ambos os períodos analisados pré e durante a pandemia, ou que estes não tiveram vendas expressivas em ambos os períodos concomitantemente. Os dados foram extraídos de sites de dados abertos do governo federal, em que são publicados dados que se referem a vendas ao consumidor de medicamentos sujeitos a escrituração junto ao Sistema Nacional de Gerenciamento Produtos Controlados (SNGPC).

Estende, temporariamente, as quantidades máximas de medicamentos sujeitos a controle especial permitidas em Notificações de Receita e Receitas de Controle.

O SNGPC monitora as compras, transferências, vendas, perdas, dentre outros, de medicamentos sujeitos a controle especial, comercializados em farmácias e drogarias privadas do país. Os dados são extraídos conforme o Art. $3^{\circ}$, da RDC $n^{\circ}$ 22/2014 (ANVISA, 2014). Todos os estabelecimentos que comercializam estes produtos devem escriturar e enviar para a Anvisa, periodicamente, os dados sobre a movimentação dos mesmos.

Os dados foram extraídos pelos autores Monique Barbosa Moreira (MBM) e confirmados por Rodrigo Inácio Novais de Oliveira (RINO). Foram consultadas as 
variáveis como: período da pesquisa, local, medicamentos analisados e suas apresentações. Em situações em que os dados não ficaram claros, os dois autores se reuniram e juntos chegaram a um consenso. Após a coleta e tabulação dos dados foi feito um teste $\mathrm{T}$ de student para diferencias as amostras pré-pandemia e durante a pandemia.

\section{RESULTADOS}

Houve o aumento na dispensação de antidepressivos, o número que no período prépandemia foi de 98.923 durante a pandemia subiu para 121.290 (Figura I). Esta alteração representa um aumento de 22,6\% (Tabela I) na cidade de Feira de Santana.

Gráfico 1. Comparativo de dispensação de medicamentos antidepressivos entre pré pandemia e pandemia em Feira de Santana - BA.

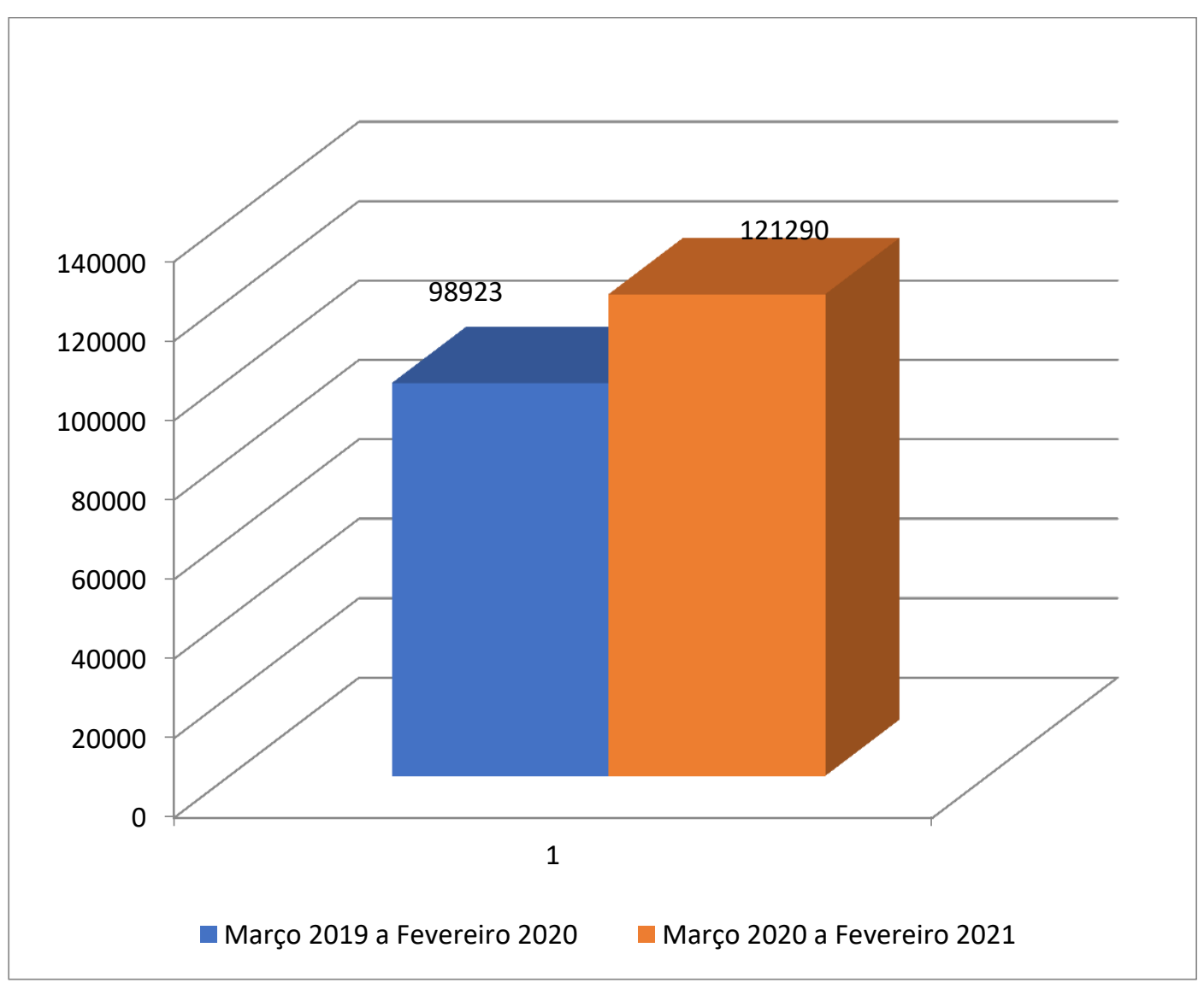


Fonte: Elaborado pelos autores a partir da revisão da literatura.

Este dado, quando comparado aos registros que representam a o Estado da Bahia (Figura 2) no mesmo período, com os mesmos medicamentos, indicam que o aumento aconteceu também em âmbito estadual, quando no período pré-pandemia o dado era de I.985.649 milhões e durante a pandemia saltou para 3.019.180 milhões, o que evidencia uma diferença de 1.033.531 milhões de medicamentos vendidos a mais. Um número bastante expressivo, este aumento equivale a $52 \%$.

Figura 2. Dispensação de medicamentos antidepressivos no estado da Bahia.

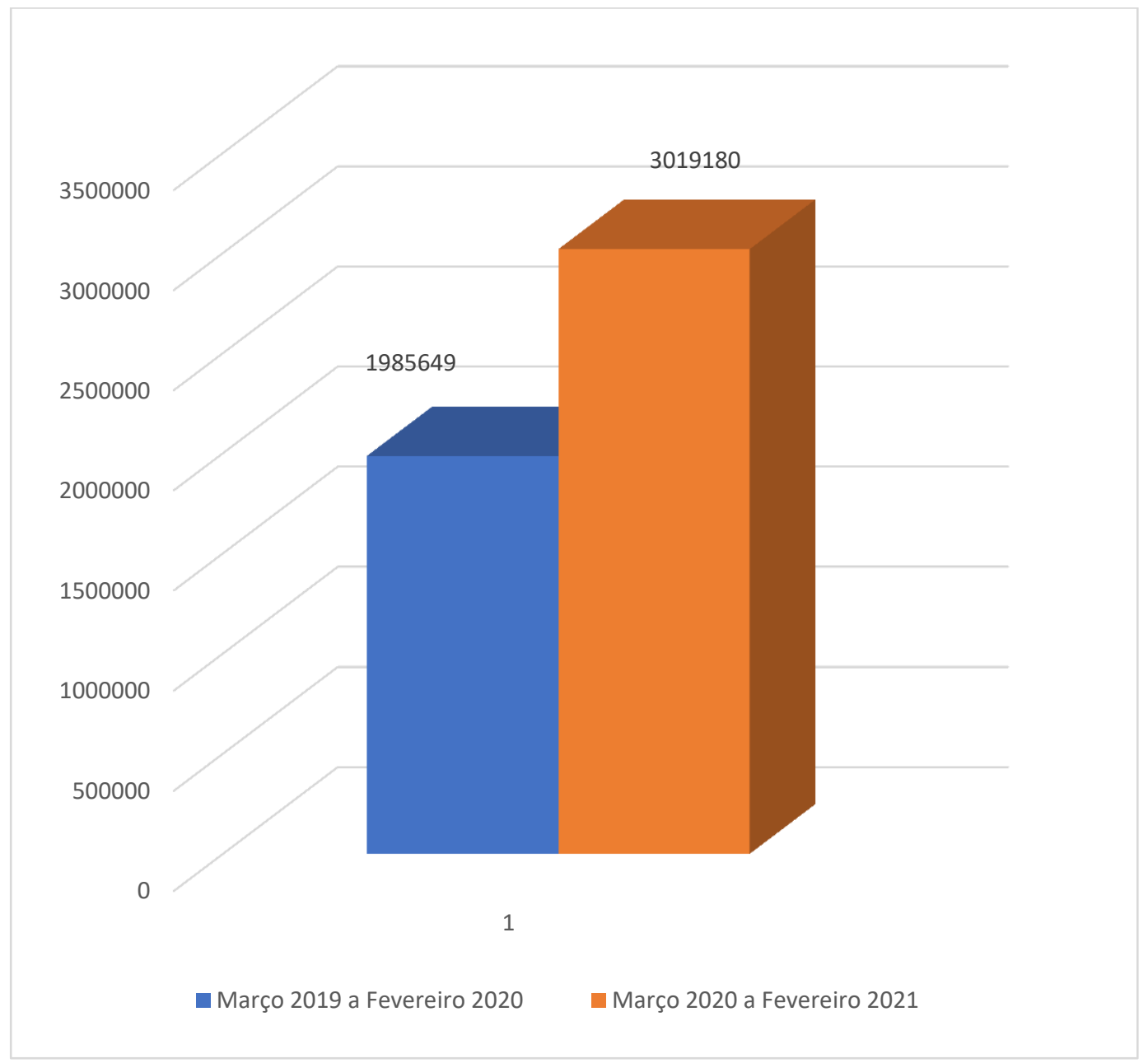

Fonte: Agência Nacional de Vigilância Sanitária 
A alteração fica mais evidente ainda quando comparamos os dados no mesmo período. Como é observado no Gráfico 3 e fica notável as dispensações de medicamentos como o Oxalato de Escitalopram que lidera com 28\% a mais do que no período prépandemia, seguido pelo Cloridrato de Amitriptilina 40\%, Cloridrato de Sertralina aumentou $36 \%$ e o Cloridrato de Fluoxetina $18 \%$, esses dados demonstram os medicamentos que já havia destaque, dispararam em dispensações durante ao período analisado.

Apesar do aumento expressivo na dispensação dos medicamentos citados acima, outros também apresentam uma diferença notória, como o Cloridrato de duloxetina que vendeu $25 \%$ a mais, o cloridrato de Venlafaxina $10 \%$, o cloridrato de trazodona 22\%, cloridrato de nortriptilina $\mathrm{\imath} 6 \%$, o citalopram $\mathrm{r} 6 \%$.

Figura 3. Comparação dos cinco medicamentos que mais sofreram variação na dispensação

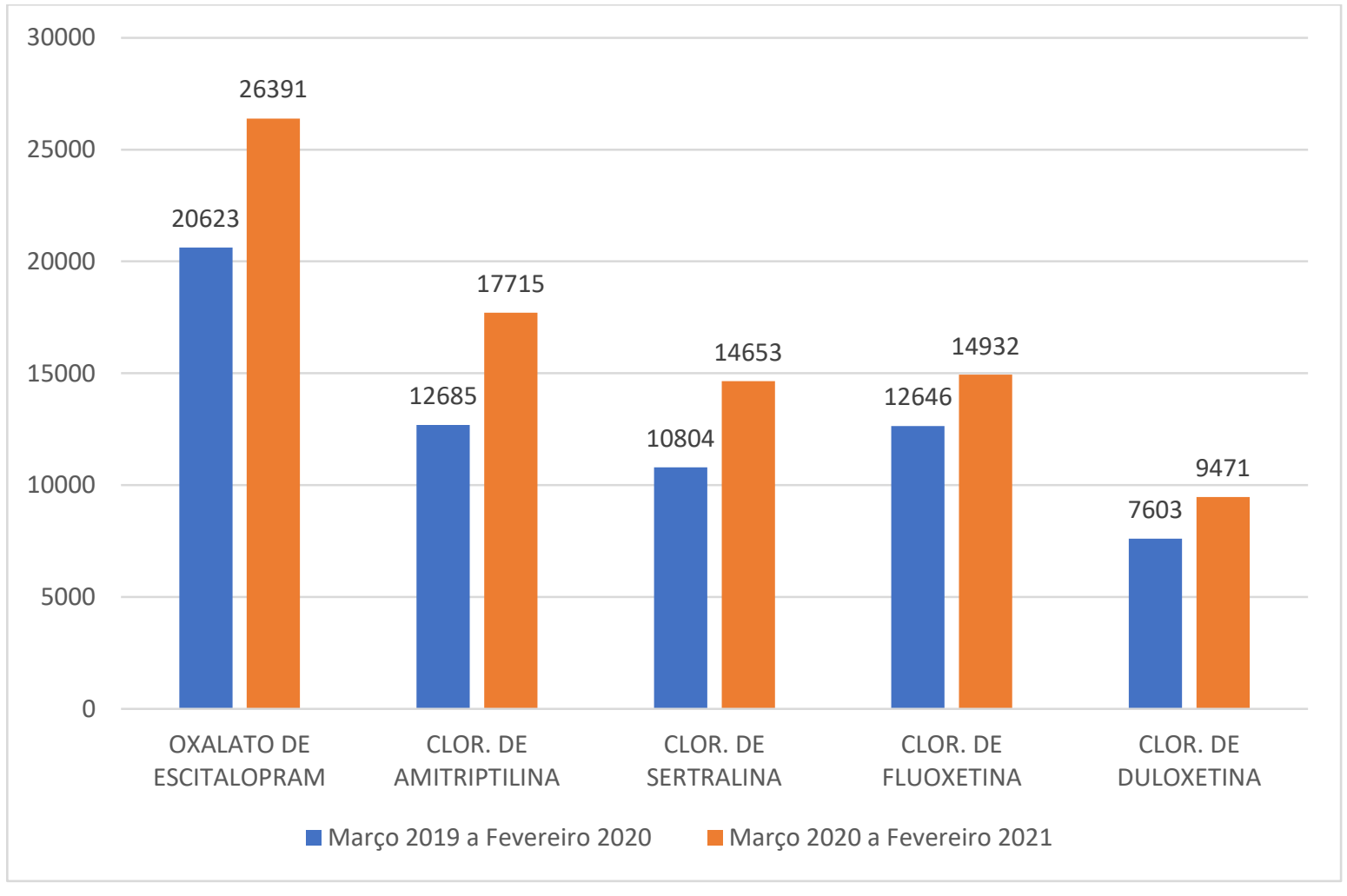

Fonte: Elaborado pelos autores a partir da revisão da literatura. 
Tabela r. Comparativo de medicamentos antidepressivos dispensados em Feira de Santana $-\mathrm{BA}$

COMPARATIVO DE MEDICAMENTOS DISPENSADOS EM FEIRA DE SANTANA - BA

\begin{tabular}{|c|c|c|c|c|c|}
\hline PRINCÍPIO ATIVO & $\begin{array}{c}\text { DISPENSAÇÃO } \\
\text { MARÇO/2019 A } \\
\text { FEVEREIRO/202 } \\
0\end{array}$ & $\begin{array}{c}\text { DISPENSAÇÃO } \\
\text { MARÇO/2020 A } \\
\text { FEVEREIRO/202 } \\
\text { I }\end{array}$ & $\begin{array}{c}\text { DIFERENÇ } \\
\text { A ENTRE } \\
\text { PERÍODOS } \\
\%\end{array}$ & $\begin{array}{c}\text { DIFERENÇA } \\
\text { EM } \\
\text { NÚMEROS } \\
\text { ABSOLUTO } \\
\text { S }\end{array}$ & \\
\hline CITALOPRAM & 4764 & 5518 & $15,8270 \%$ & 754 & \\
\hline $\begin{array}{c}\text { CLORDIAZEPÓXID } \\
\text { O + CLOR. DE } \\
\text { AMITRIPTILINA }\end{array}$ & 1879 & 1945 & $3,5125 \%$ & 66 & \\
\hline $\begin{array}{c}\text { CLOR. DE } \\
\text { AMITRIPTILINA }\end{array}$ & 12685 & 17715 & $39,6531 \%$ & 5030 & \\
\hline $\begin{array}{l}\text { CLOR. DE } \\
\text { BUPROPIONA }\end{array}$ & 4052 & 4389 & $8,3169 \%$ & 337 & \\
\hline $\begin{array}{l}\text { CLOR. DE } \\
\text { CLOMIPRAMINA }\end{array}$ & 2165 & 2166 & $0,0462 \%$ & I & \\
\hline $\begin{array}{c}\text { CLOR. DE } \\
\text { DULOXETINA }\end{array}$ & 7603 & 9471 & $24,5692 \%$ & I868 & \\
\hline $\begin{array}{c}\text { CLOR. DE } \\
\text { FLUOXETINA }\end{array}$ & 12646 & 14932 & І8,0769\% & 2286 & 3186 \\
\hline $\begin{array}{l}\text { CLOR. DE } \\
\text { IMIPRAMINA }\end{array}$ & 643 & 132 & $-79,4712 \%$ & $-5 \mathrm{II}$ & \\
\hline $\begin{array}{c}\text { CLOR. DE } \\
\text { NORTRIPTILINA }\end{array}$ & 4636 & 5365 & $15,7248 \%$ & 729 & \\
\hline $\begin{array}{c}\text { CLOR. DE } \\
\text { SERTRALINA }\end{array}$ & 10804 & 14653 & $35,6257 \%$ & 3849 & \\
\hline $\begin{array}{c}\text { CLOR. DE } \\
\text { TRAZODONA }\end{array}$ & 4002 & 4854 & $21,2894 \%$ & 852 & \\
\hline $\begin{array}{c}\text { CLOR. DE } \\
\text { VENLAFAXINA }\end{array}$ & 7126 & 7820 & $9,7390 \%$ & 694 & \\
\hline $\begin{array}{l}\text { HIDROBROMETO } \\
\text { DE CITALOPRAM }\end{array}$ & 20 & 13 & $-35,0000 \%$ & -7 & \\
\hline $\begin{array}{l}\text { MALEATO DE } \\
\text { FLUVOXAMINA }\end{array}$ & 813 & 917 & $\mathrm{I} 2,7921 \%$ & 104 & \\
\hline MIRTAZAPINA & 4457 & 4978 & II,6895\% & 521 & \\
\hline $\begin{array}{l}\text { OXALATO DE } \\
\text { ESCITALOPRAM }\end{array}$ & 20623 & 26391 & $27,9688 \%$ & 5768 & \\
\hline $\begin{array}{c}\text { SULFATO DE } \\
\text { TRANILCIPROMIN } \\
\text { A }\end{array}$ & 5 & $3 \mathrm{I}$ & $520,0000 \%$ & 26 & \\
\hline TOTAL & 98923 & 121290 & $22,6105 \%$ & 22367 & \\
\hline
\end{tabular}


$\mathrm{O}$ teste $\mathrm{T}$ devolveu o valor de $\mathrm{P}=0,005123965$ evidenciando uma variação significativa (Abaixo de 5\% de significância) entre as amostras coletadas no período pré pandemia e durante a pandemia.

\section{DISCUSSÃO}

A dispensação de ansiolíticos benzodiazepínicos conhecidos como (BDZ), se destacou nos períodos consultados como fármacos de controle para o tratamento da ansiedade e depressão. (AZEVEDO, 2016)

Em conformidade com o Sistema Nacional de Vigilância Sanitária (ANVISA) através do programa de Sistema Nacional de Gerenciamento de Produtos controlados (SNGPC), verificamos informações dos lançamentos de receitas de controle especial, que é obrigatório as Farmácias e Drogarias realizar o lançamento das vendas dos medicamentos acólitos no sistema de gerenciamento, assim podemos relacionar se houve um aumento ou não dos ansiolíticos. (AZEVEDO, 2016)

Os diversos dados obtidos através da pesquisa permitem a observação do aumento na dispensação de antidepressivos em vários aspectos.

Inicialmente, vale elucidar que segundo a Organização Mundial da Saúde ${ }^{\mathrm{I}}$ que a depressão é um transtorno mental comum que apresenta humor deprimido, perda de interesse ou prazer, diminuição da energia, sentimento de culpa ou baixo auto-estima, sono ou apetite perturbado e pobre concentração.

A coleta de análise de dados dos medicamentos ansiolíticos e demais substâncias de controle especial, permitem analisar a variação e relevância entre o consumo dos ansiolíticos de determinados locais sendo possível relacionar regiões desejadas (BRASIL, I998)

O tratamento da depressão envolve o uso de medicamentos sujeitos a controle especial. A psicoterapia auxilia o paciente, mas não evita novos eventos, nem cura a depressão. $O$ método auxilia na reestruturação psicológica do indivíduo, além de elevar seu conhecimento sobre o processo de depressão e na resolução de conflitos, o que diminui o impacto provocado pelo estresse. De acordo o sanitarista Paulo 
Amarante, em uma entrevista dada ao blog do CEE/Fiocruz, a terapia com fármacos desta classe, pode causar uma certa dependência e, na maioria das vezes, a dose precisa ser reajustada (POWELL, 2008).

Como não se sabe ao certo quando será o fim da pandemia, o fim do uso obrigatório de máscaras e, principalmente, quando será livre a opção de continuar ou não em isolamento, tais pessoas que iniciaram um tratamento desse tipo, não têm prazo para encerrá-lo, sendo conhecidos fatos de uso contínuo desses medicamentos. Além do medo de adquirir a doença e não saber ao certo - no início da pandemia - o que o vírus poderia provocar no organismo antes de evoluir ao óbito, o pavor de morrer em decorrência da falta de insumos alimentares para si e sua família era grande, pois o trabalhador foi obrigado a não sair de sua residência, sendo assim não garantiria o sustento para os seus, desta forma seu fim poderia ser morrer de fome ou do vírus. A depressão já vinha sendo destaque, pois segundo o Ministério da Saúde entre 2015 e 2018 houve um aumento de 52\% de atendimentos no SUS relacionados a depressão. (SILVA, 2020).

Atualmente, considerando a pandemia e o aumento na dispensação dos medicamentos antidepressivos esses dados estão mais elevados.

A pandemia desenvolve uma pressão psicológica insuportável, aumentando
a possibilidade de problemas psicológicos e mentais, principalmente devido
à quarentena que distancia, gradualmente, as pessoas umas das outras. Desse
modo, observa-se a grande necessidade de cuidados direcionados à saúde mental
durante o surto de COVID-I9. A qualidade do sono tem sido apontada como
um indicador-chave." "Uma pesquisa em um hospital de Hong Kong
mostrou que, aproximadamente, 50\% dos pacientes com SARS
recuperados apresentaram ansiedade, e, aproximadamente, 20\% estavam com
medo. Inclusive, 20\% dos pacientes reabilitados apresentaram alguns efeitos
psicológicos e psiquiátricos negativos, incluindo insônia e depressão. (VIANA,
2019)

O que justifica o crescimento na dispensação dos medicamentos para tratar a depressão. Segundo Goodman:

Os medicamentos comumente usados, muitas vezes, chamados de antidepressivos de segunda geração, são os inibidores seletivos de recaptação da serotonina (ISRSs) e os inibidores $\mathrm{da}$ recaptação de serotonina-norepinefrina (IRSNs), que tem maior eficácia e segurança em relação aos fármacos de primeira geração que incluem os inibidores da monoaminoxidase (MAO) e os antidepressivos tricíclicos (ATC). (BRUNTON,2018, p.239) 
O manual de farmacoterapia de Wells (2016), mostra que "entre 65 e 70\% dos pacientes com depressão melhoram com a terapia farmacológica."

Em 1988, uma nova classe de antidepressivos surgiu, inaugurada, inicialmente, pela fluoxetina e seguida por vários outros: paroxetina, sertralina, citalopram e fluvoxamina. Esses fármacos apresentam vantagens em relação aos antidepressivos convencionais: suas meias- vidas prolongadas permitem administração em dose única diária, contribuindo assim para maior adesão ao tratamento" (SILVA, 2oro p. 348)

Seu mecanismo de ação se explica por “os ISRS possuem ação seletiva bloqueando a recaptação de serotonina nos receptores 5-HTI, 5-HTI e 5-HT3, aumentando a concentração de serotonina na fenda sináptica.” (SILVA 2010, p.350).

Conforme foi analisado nos dados (Gráfico 4), parte dos medicamentos que mais cresceram pertencem a classe dos antidepressivos, como é o caso da fluoxetina, sertralina, escitalopram. E como é citado na própria bula do cloridrato de sertralina ela é "eficaz tanto na prevenção de recaída dos sintomas do episódio inicial de depressão, assim como na recorrência de outros episódios depressivos." (2019)

$\mathrm{O}$ uso destes medicamentos pode levar a efeitos colaterais que merecem destaque, como efeito estimulante do Sistema Nervoso Central (SNC) e diarreia, vômito e náusea. (SILVA, 2010 p. 350).

A venlafaxina é um antidepressivo seguro e com baixo poder de interação medicamentosa. Quando administrada em pacientes com insuficiência renal e hepática, suas doses devem ser reduzidas. Em casos de suspensão do tratamento, sua retirada deve ser gradativa, pois pode acarretar a síndrome de descontinuação, caracterizada por náusea, tontura, mal-estar e sudorese, que pode persistir por até zo dias após sua suspensão. (SILVA, 2010, p. 351)

As classes de antidepressivos inibidores da monoaminoxidase (MAO) e os antidepressivos tricíclicos (ATC) também conhecidos como fármacos de primeira geração, tem como mecanismo de ação segundo Goodman:

Aumentam o armazenamento do neurotransmissor nos grânulos secretores, os ADT inibem a captação de $5-\mathrm{HT}$ e a norepinefrina. Embora eficazes, esses agentes de primeira geração apresentam efeitos colaterais e interações medicamentosas e alimentares que limitam a sua utilização relativamente aos antidepressivos mais recentes. (BRUNTON, 2018, p 398)

O que se deve levar em consideração para manutenção e otimização dos resultados da farmacoterapia, são os efeitos colaterais que estes medicamentos trazem, ainda de acordo com Goodman:

Mediados pelo sistema nervoso parassimpático (visão turva, boca seca, taquicardia, constipação, dificuldade para urinar). Ocorre uma determinada 
tolerância para estes efeitos anticolinérgicos, que são mitigados por meio de estratégias de titulação para atingir doses terapêuticas durante um período razoável. $O$ antagonismo de receptores a $\mathrm{I}$-adrenérgicos contribui para a hipotensão ortostática e sedação. $O$ ganho de peso é outro efeito colateral desta classe de antidepressivos (BRUNTON, 20I8, p. 4II)

Diante dos efeitos colaterais e adversos que estes fármacos causam é importante destacar o motivo pelo qual está terapia foi necessária. Logo é destacado as mudanças de estilo de vida que foram impostas pela pandemia de forma abrupta e quais os impactos que foram causados no psicológico de todos.

A pandemia do coronavírus impactou a sociedade e todos os indivíduos em diversas esferas. Primeiramente, houve um grande impacto no âmbito financeiro, uma vez que empreendimentos, comércios e outros tipos de negócios tiveram que ser interrompidos, o que resultou na perda de empregos e falência de empresas. O desemprego ou a diminuição da renda são fatores ligados ao surgimento de sintomas depressivos e ansiosos, devido à incerteza e o medo de não conseguir arcar com as responsabilidades mensais e necessidade dos familiares. Além disso, foi necessário promover o isolamento social para diminuir a propagação do vírus e o número de casos." Foi o que falou Dra. Luciana Mancini Bari, médica e Antônio Chaves, psicólogo do Hospital Santa Mônica, ainda segundo a Dra. Luciana, "para algumas pessoas essa medida significou um grande sofrimento, visto que muitas moram sozinhas, ficando sem nenhuma companhia durante a quarentena, ou se preocupam com entes queridos mais sensíveis que estão longe, como os idosos e pessoas com deficiência ou distúrbios psíquicos. Há, também, o medo de ser contaminado pelo vírus e sofrer as consequências da doença. $O$ número de mortos pelo coronavírus despertou um grande alerta na população, que teme principalmente pelos mais velhos e pessoas com doenças pré-existentes." (Hospital Santa Mônica, 2020)

Ainda podemos destacar públicos distintos e suas vivências diante do novo formato de vida tão distinta e seu impacto nas convivências em grupo, como exemplo disso é:

As mulheres e os idosos que têm ocupação com exposição ao COVID- I9 apresentaram maiores escores de depressão e as pessoas casadas valores menores. Não ter graduação esteve associado à uma maior frequência entre ter sintomas depressivos; por outro lado, receber cinco salários, ou mais, diminuiu as chances dessa sintomatologia” $O$ que traça o perfil dos casos de depressão durante a pandemia. (PEREIRA-AVILA, 202I)

Perante a necessidade desta terapia é imprescindível que este tratamento tenha o acompanhamento farmacoterapêutico, visando a adesão do paciente, a continuidade da farmacoterapia e o acompanhamento farmacêutico para sanar dúvidas e queixas do paciente. 
Para que a farmacoterapia promova algum efeito terapêutico, é necessário o transcurso do período de pelo menos duas semanas, conhecido como período de latência (adaptações farmacodinâmicas que resultam na redução do número e da afinidade dos receptores ao longo do tempo, necessárias para promoção do efeito farmacológico). Destaca-se ainda que, para que ocorra alguma resposta clínica e a redução expressiva dos sintomas, os antidepressivos devem ser utilizados por pelo menos quatro semanas. Além disso, é necessário atingir as doses terapêuticas sem ultrapassar as doses toleradas pelo paciente e garantir a adesão ao tratamento. É importante ainda lembrar que o tratamento farmacológico deve ser orientado por profissionais de saúde (no caso, médico e farmacêutico) e que deve ser individualizado para cada paciente. (SILVA, A.S, 2021, p.03)

O papel do farmacêutico na dispensação e orientação aos pacientes que utilizam esses medicamentos, é de fundamental importância uma vez que:

As atividades desenvolvidas pelos farmacêuticos em proporcionar orientação, conhecimento sobre os eventos adversos, uso correto e seguro dos antidepressivos além de outras informações a respeito do tratamento, podem repercutir no aumento da confiança recuperação da doença tornando o processo de cuidar da saúde mais seguro e humanizado" (SOUZA, M.A.; TREVISAN M. A. 2021, p.o6)

\section{CONCLUSÃO}

Diante dos dados colhidos e apresentados é notável o aumento na dispensação de antidepressivos durante a pandemia de covid-ı́ na cidade de Feira de Santana e na Bahia, ainda é perceptível os motivos que levaram a este aumento, como a insegurança que o desemprego gerado pelo lockdown em que muitas empresas não conseguiram se manter, o medo da doença desconhecida e as incertezas para o futuro trazidas pela pandemia de covid-ı́ a uma população que não sabia lidar com tamanha crise de saúde pública. Esse estudo abre oportunidade a novas pesquisas a respeito da depressão e antidepressivos no que diz respeito a suas alterações durante a pandemia e até sobre as consequências dela para a convivência humana social e quais serão os rumos que este problema trará no futuro para a humanidade.

\section{REFERÊNCIAS}

Weekly Operational Update on COVID-r9. World Health Organization. Disponível em: 〈https://www.who.int/emergencies/diseases/novel-coronavirus-2019/situation-reports.〉 Acesso em: 12 de Nov. 202I

COLTRI, F. Antidepressivos de inibidores seletivos são os mais usados. Jornal da USP, 
2019, Disponível em: 〈https://jornal.usp.br/atualidades/antidepressivos-de-inibidoresseletivos-sao-os-mais-usados/>. Acesso em: I2 de Nov. 2021

JÚNIOR, A. G. C. et. al. Reações autonômicas e hormonais das perturbações psicossomáticas. Revista de Psicofisiologia. Vol.2, n.I, 1998. Disponível em: <http://labs.icb.ufmg.br/lpf/mono5.pdf.> Acesso em: io de Nov. 202I

Relatório mundial da saúde: saúde mental: nova concepção, nova esperança. ORGANIZAÇÃO MUNDIAL DA SAÚDE. 2002 Disponível em: < https://www.who.int/whr/200I/en/whroI_po.pdf> Acesso em Is de Mar. 2021

GUIMARÃES C. Corona vírus: atendimentos psiquiátricos crescem $25 \%$ com a pandemia. 2020. Veja. Disponível em: <https://vejario.abril.com.br/coronavirus/coronavirus- atendimentos-psiquiatricosaumentam-pandemia/>. Acesso em: I2 de Nov. 202I

SCHEINKMAN L. Ansiedade e depressão em sobreviventes da COVID-ı: Papel dos preditores inflamatórios e clínicos. Instituto Brasileiro de Informação em Ciência e Tecnologia. 2021. Disponível em: http://evidenciascovidı.ibict.br/index.php/tag/transtorno-de-estresse-pos-traumatico/.

Acesso em: I6 de Nov. 202I.

Secretaria de Saúde confirma Io caso de coronavírus na Bahia. Gi. 2021. Disponível em: https://gr.globo.com/ba/bahia/noticia/2020/o3/o6/secretaria-de-saude-confirma-ıcaso-de-coronavirus-na-bahia.ghtml. Acesso em: I2 de Nov. 202I

HILAL-DANDAN, R.; BRUNTON, L. Manual de Farmacologia e Terapêutica de Goodman \& Gilman. 2nd ed. Brasil: AMGH; 2014.

ANVISA. Resolução RDC $\mathrm{n}^{\mathrm{o}}$ 22, de 29 de abril de 2014. Disponível em: <http://portal.crfsp.org.br/325-legislacao/5508-resolucao-rdc-22-de-29-de-abril-de-2014anvisa.html> Acesso em: 8 de Nov. 2021.

AZEVEDO, A.J.P, ARAÚJO, A.A., FERREIR, M.A.F. Consumo de ansiolíticos benzodiazepínicos: uma correlação entre dados do SNGPC e indicadores sociodemográficos nas capitais brasileiras. Ciênc. saúde colet. v. 2I n.I, 20I6. Disponível em: $\langle$ https://www.scielo.br/j/csc/a/LZdp4JrmHzn6XbXff 4 TVpyN/?lang=pt\#17> Acesso em: I2 de Novembro.

BRASIL, Ministério da Saúde, Secretaria de Vigilância em Saúde, PORTARIA № 344, DE 12 DE MAIO DE 1998. Secretaria de Vigilância em Saúde; 1998 Disponível em: < https://bvsms.saude.gov.br/bvs/saudelegis/svs/1998/prt0344_I2_05_1998_rep.html>. Acesso em: or de Nov. 202I. 
POWELL, V.B. et al. Terapia cognitivo-comportamental da depressão. Revista Brasileira de Psiquiatria. v.30, n.02 2008 Disponível 〈https://www.scielo.br/j/rbp/a/XD $3 \mathrm{XXdNxQPMwj6gc4}_{4} \mathrm{WRjqSB} /$ ?lang=pt> Acesso em: 3I de Out. 202I

SILVA J.K. et al. A relação entre a infecção por coronavírus e susceptibilidade a transtornos mentais e o risco de suicídio: o que a literatura tem evidenciado?. J. Health Biol Sci. 2020; v.8 n.or Disponível em: 〈https://pesquisa.bvsalud.org/portal/resource/pt/biblio-IIooos6〉. Acesso em: Is de Março de 2021

VIANA, R. Atendimentos do SUS a jovens com depressão crescem II5\% em três anos. GI. 2019. Disponível em: < https://gi.globo.com/bemestar/depressao/noticia/2019/o9/17/atendimentos-do-sus-ajovens-com-depressao-crescem-IIspercent-em-tres-anos.ghtml2or9>. Acesso em: i7 de setembro de 2021.

BRUNTON, L.; HILAL-DANDAN R; KNOLLMANN, B.C. As Bases Farmacológicas da Terapêutica de Goodman e Gilman. Porto Alegre: 13th ed. AMGH Editora Ltda, 2018.

WELLS, B. Manual de Farmacoterapia. Porto Alegre: 9th ed. Estudio Costellani, 2016.

SILVA, P. Farmacologia. Rio de Janeiro. 8. Ed, Guanabar. 2010.

CLORIDRATO DE SERTRALINA: comprimido. Responsável Técnico: Alexandre Canellas de Souza. Ahmedabad 382 210 - Índia. CADILA HEALTHCARE LIMITED, 2019. I bula de remédio $(23 \mathrm{p})$. Bula. Disponível em: <https://bulas.postosdesaude.com.br/pdfs/merck_s_a_cloridrato_de_sertralina_profission al.pdf> Acesso em io Nov. 2021.

Ansiedade e depressão na pandemia: entenda o crescimento nos casos e como pedir ajuda. Hospital Santa Mônica, 2020; Disponível em: < https://hospitalsantamonica.com.br/ansiedade-e-depressao-na-pandemia/>. Acesso em io de Nov. 2021.

PEREIRA-AVILA, F.M.V. et. al. Fatores Associados aos Sintomas de Depressão entre Idosos Durante a Pandemia da Covid -i9. Texto e Contexto Enfermagem v. 30. n.I 202I. Disponível em: <https://www.scielo.br/j/tce/a/4y7pZxLbhnwkssDnczhxrMf/?lang=pt\#> Acessado em: io de Nov. 2021.

SILVA, A.S et. al. Antidepressivos no Transtorno Depressivo Maior em Adultos. Boletim Brasileiro de Avaliação de Tecnologias em Saúde. v.I8. 2021. Disponível em: 〈https://bvsms.saude.gov.br/bvs/periodicos/brats_i8.pdfor >. Acessado em: io de Nov. 2010. 
SOUZA, M.A.; TREVISAN M. A. Depressão no idoso e o papel do farmacêutico na terapia medicamentosa. Revista Artigos.com. v.28, 2021 May or Disponível em: <https://acervomais.com.br/index.php/artigos/issue/view/21>. Acessado em is de Mar. 2021. 